\title{
Clinically-relevant consecutive treatment with isoproterenol and adenosine protects the failing heart against ischaemia and reperfusion
}

\author{
Igor Khaliulin", Andrew P Halestrap, Simon M Bryant, Declan J Dudley, Andrew F James and M-Saadeh Suleiman
}

\begin{abstract}
Background: Consecutive treatment of normal heart with a high dose of isoproterenol and adenosine (Iso/Ade treatment), confers strong protection against ischaemia/reperfusion injury. In preparation for translation of this cardioprotective strategy into clinical practice during heart surgery, we further optimised conditions for this intervention using a clinically-relevant dose of Iso and determined its cardioprotective efficacy in hearts isolated from a model of surgically-induced heart failure.

Methods: Isolated Langendorff-perfused rat hearts were treated sequentially with $5 \mathrm{nM}$ Iso and $30 \mu \mathrm{M}$ Ade followed by different durations of washout prior to $30 \mathrm{~min}$ global ischaemia and 2 hrs reperfusion. Reperfusion injury was assessed by measuring haemodynamic function, lactate dehydrogenase (LDH) release and infarct size. Protein kinase C (PKC) activity and glycogen content were measured in hearts after the treatment. In a separate group of hearts, Cyclosporine A (CSA), a mitochondria permeability transition pore (MPTP) inhibitor, was added with Iso/Ade. Failing hearts extracted after 16 weeks of ligation of left coronary artery in 2 months old rats were also subjected to Iso/Ade treatment followed by ischaemia/reperfusion.
\end{abstract}

Results: Recovery of the rate pressure product (RPP) in Iso/Ade-treated hearts was significantly higher than in controls. Thus in Iso/Ade treated hearts with $5 \mathrm{nM}$ Iso and no washout period, RPP recovery was $76.3 \pm 6.9 \%$ of initial value vs. $28.5 \pm 5.2 \%$ in controls. This was associated with a 3 fold reduction in $\mathrm{LDH}$ release irrespective to the duration of the washout period. Hearts with no washout of the drugs (Ade) had least infarct size, highest PKC activity and also showed reduced glycogen content. Cardioprotection with CsA was not additive to the effect of Iso/Ade treatment. Iso/Ade treatment conferred significant protection to failing hearts. Thus, RPP recovery in failing hearts subjected to the treatment was $69.0 \pm 16.3 \%$ while in Control hearts $19.7 \pm 4.0 \%$. LDH release in these hearts was also 3 fold lower compared to Control.

Conclusions: Consecutive Iso/Ade treatment of normal heart can be effective at clinically-relevant doses and this effect appears to be mediated by glycogen depletion and inhibition of MPTP. This intervention protects clinically relevant failing heart model making it a promising candidate for clinical use.

Keywords: Ischaemia/reperfusion, Cardioprotection, Isoproterenol, Adenosine, Heart failure, Mitochondria permeability transition pore

\footnotetext{
* Correspondence: i.khaliulin@bristol.ac.uk

School of Clinical Sciences and Bristol Cardiovascular, University of Bristol,

Bristol Royal Infirmary, Upper Maudlin Street, Bristol BS2 8HW, UK
}

(c) 2014 Khaliulin et al.; licensee BioMed Central Ltd. This is an Open Access article distributed under the terms of the Creative Commons Attribution License (http://creativecommons.org/licenses/by/4.0), which permits unrestricted use, distribution, and reproduction in any medium, provided the original work is properly credited. The Creative Commons Public Domain Dedication waiver (http://creativecommons.org/publicdomain/zero/1.0/) applies to the data made available in this article, unless otherwise stated. 


\section{Background}

Reperfusion following a prolonged period of ischaemia induces myocardial dysfunction and necrotic damage [1]. Ischaemia and reperfusion-elicited tissue injury contributes to morbidity and mortality in a wide range of pathologies and is a major challenge during organ transplantation and cardiothoracic, vascular and general surgery [2].

We have previously described a cardioprotective protocol termed temperature preconditioning (TP) [3] where a few brief episodes of hypothermic perfusion $\left(\right.$ at $26^{\circ} \mathrm{C}$ ) interspersed with normothermic perfusion $\left(\right.$ at $37^{\circ} \mathrm{C}$ ) significantly improved heart resistance to the subsequent index ischaemia. The cardioprotective effect of TP, which exceeds the effect of ischaemic preconditioning, is associated with activation of protein kinases $\mathrm{A}$ (PKA) and $\mathrm{C}$ (PKC) and can be abolished by inhibitors of PKA and PKC (H-89 and chelerythrine, respectively) $[3,4]$. Furthermore, a combination of TP with the PKA inhibitor H-89 (which has no direct effect on $\mathrm{PKC}$ ) reduced activation of $\mathrm{PKC}$ indicating that in the mechanism of TP, PKC activation occurs downstream to activation of PKA [4]. Importantly, TP mediated by the consecutive activation of PKA and PKC protects hearts during global normothermic ischaemia and during hypothermic ischaemia and cardioplegia [5]. When the consecutive activation of PKA and PKC was mimicked pharmacologically using the PKA activator isoproterenol (Iso) and the PKC activator adenosine (Ade), it resulted in a highly potent cardioprotective effect [4]. This was achieved using a concentration of Iso (200 nM) significantly higher than the concentration of isoproterenol in blood plasma used clinically (0.02-0.2 $\mu \mathrm{g} / \mathrm{kg} / \mathrm{min}$; equivalent to about $1.3-13 \mathrm{nM})$ [6]. In contrast, the concentration of adenosine used in our experiments $(30 \mu \mathrm{M})$ is considerably lower than that used for blood cardioplegia during cardiac surgery $(1-2 \mathrm{mM})[7,8]$. Therefore it is important to test both drugs at concentrations that are clinically relevant. Furthermore, in our earlier work investigating this intervention, we had not explored what duration of the washout period prior to ischaemia produced the maximal cardioprotective effect by triggering signalling mechanisms.

It is now recognized that opening of the mitochondria permeability transition pore (MPTP) plays a key role in mediating the irreversible damage of the myocardium during reperfusion [9] and that a range of interventions that inhibit pore opening, either directly or indirectly, are cardioprotective [10-12]. This was confirmed for the cardioprotective effect of consecutive Iso/Ade treatment with Iso used at relatively high concentration [4] but has not been investigated at a clinically relevant concentration. This can be achieved by establishing whether the cardioprotective effects of Iso/Ade treatment are additive to those of cyclosporine A (CsA) which is one of the most effective inhibitors of MPTP [13] widely used in experiments on isolated hearts.
The presence of co-morbid illness can render the myocardium resistant to cardioprotection against infarction by physical or pharmacological stimuli [14]. Thus an important and necessary step towards translation of this cardioprotective strategy into clinical practice is to study the efficacy of consecutive Iso/Ade treatment in diseased hearts.

Here we study whether our treatment is effective at low, clinically relevant concentrations of Iso and optimize the protocol of the consecutive Iso/Ade treatment. We confirm the underlying mechanism to be inhibition of MPTP opening and that the treatment is effective in a surgicallyinduced model of heart failure.

\section{Methods}

Unless otherwise stated, all biochemicals were purchased from Sigma and general chemicals from Fischer Scientific or VWR-Jencons.

\section{Experimental Procedures}

\section{Heart perfusion and analysis of haemodynamic function}

All procedures conform to the Directive 2010/63/EU of the European Parliament and the Guide for the Care and Use of Laboratory Animals published by the US National Institutes of Health (NIH Publication No. 85-23, revised 1996). Ethical approval was granted by the University of Bristol, UK. Two months old male Wistar rats (250-260 g) were killed by stunning and cervical dislocation. Hearts $(\sim 0.75 \mathrm{~g})$ were rapidly removed into ice-cold Krebs-Henseleit buffer $(\mathrm{KH})$ containing $(\mathrm{mM}): \mathrm{NaCl} 118, \mathrm{NaHCO}_{3} 25, \mathrm{KCl} 4.8$, $\mathrm{KH}_{2} \mathrm{PO}_{4} 1.2, \mathrm{MgSO}_{4} 1.2$, glucose 11 and $\mathrm{CaCl}_{2} 1.2$, gassed with $95 \% \mathrm{O}_{2}-5 \% \mathrm{CO}_{2}$ at $37{ }^{\circ} \mathrm{C}(\mathrm{pH}$ 7.4) and perfused in Langendorff mode. The aorta was rapidly cannulated and the heart perfused at a rate of $8 \mathrm{ml} \cdot \mathrm{min}^{-1} \cdot \mathrm{g}^{-1}$ heart weight with in-line filter using $\mathrm{KH}$. Monitoring of left ventricular pressure was performed with a water-filled balloon inserted into the left ventricle, set to give an initial left ventricular end diastolic pressure (LVEDP) of $2.5-5 \mathrm{mmHg}$. All hearts were allowed an equilibration period of at least $25 \mathrm{~min}$ before any additional treatments was given. Data acquisition and analysis used a PowerLab System (ADInstruments, Australia). Left ventricular developed pressure (LVDP) was calculated as the difference between left ventricular systolic pressure (LVSP) and LVEDP, and work index (RPP) as the product of LVDP and heart rate (HR). Time derivatives of pressure were measured during contraction $(+\mathrm{dP} / \mathrm{dt})$ and relaxation $(-\mathrm{dP} / \mathrm{dt})$. The haemodynamic parameters were computed using the software Chart 5 (ADInstruments, Australia). Preischaemic values of the parameters of haemodynamic function were measured at the end of the equilibration period prior to any preischaemic intervention. Since the hearts in our experiments contracted spontaneously, haemodynamic function is reflected in both LVDP and HR. For this reason, 
RPP was considered as the main parameter characterising haemodynamic function. If RPP was less than $15,000 \mathrm{mmHg} \cdot$ beat $/ \mathrm{min}$ at the end of the preischaemic equilibration period, the heart was excluded from the experiment.

\section{Model of Surgically-Induced Heart Failure}

Heart failure was surgically induced by ligation of the left coronary artery as described previously [15] and modified in our laboratory. In brief, 2 month old rats were anesthetized with ketamine $(75 \mathrm{mg} / \mathrm{kg}$, i.p.) and medetomidine ( $0.5 \mathrm{mg} / \mathrm{kg}$, i.p.). Analgesia was induced by buprenorphine $(0.05 \mathrm{mg} / \mathrm{kg}$, s.c.). The rats were intubated and ventilated with a respirator for small animals (Harvard Apparatus). Body temperature was maintained at $37^{\circ} \mathrm{C}$ during the surgical procedure. An incision was made in the left side of the chest to expose the fourth intercostal space. A 7-0 surgical suture was placed under the left coronary artery and tied to complete occlusion of the artery. In shamoperated animals (SO), the coronary artery was not ligated. The wound was closed with a 4-0 surgical suture. Atipamezole $(2.5 \mathrm{mg} / \mathrm{kg}$, i.p.) was injected in order to facilitate recovery after the anaesthesia. After the surgery, rats were placed into a recovery chamber (Vet Tech Solutions Ltd) ventilated with oxygen. In coronary artery ligated (CAL) animals, approximately 92\% survived and were able to meet the inclusion criteria for the experiments on isolated heart. All sham operated animals survived to 16 weeks after surgery. Animals were monitored and cared for daily. Sixteen weeks after surgery, the animals were used for experiments on isolated heart as shown in the Methods.

\section{Experimental Groups}

After a 25 min period of equilibration followed by preischaemic interventions, global normothermic ischaemia $\left(37^{\circ} \mathrm{C}\right)$ was induced for $30 \mathrm{~min}$ and then normothermic perfusion was reinstated for $2 \mathrm{hr}$. Three series of experiments were performed as detailed below and summarised in Figures 1 and 2. In Series 1 and 2, experiments were carried out on healthy male Wistar rats (250-275 g) whilst in Series 3 rats with surgically-induced heart failure or sham-operated rats were employed.

In Series 1 (Figure 1A), hearts were divided into a Control group and 4 treatment groups (8-10 hearts/ group) according to the preischaemic protocol. Hearts of the Control group were not subjected to any intervention prior to ischaemia. Hearts of the three treatment groups, after the equilibration period, were subjected to the consecutive perfusion with Iso and Ade ( $3 \mathrm{~min}$ with $5 \mathrm{nM}$ Iso and $5 \mathrm{~min}$ with $30 \mu \mathrm{M}$ Ade) followed by a washout period of different duration: $10 \mathrm{~min}$ (Iso/Ade10); $5 \mathrm{~min}$ (Iso/Ade-5) and zero min (Iso/Ade-0). Hearts of the fourth treatment group ((Iso/Ade Mix)) were perfused with Ade for $1.5 \mathrm{~min}$ and then $3 \mathrm{~min}$ with the mixture of Iso and Ade followed by another $1.5 \mathrm{~min}$ period of perfusion with Ade (Figure 1A). Analysis of haemodynamic function recovery, lactate dehydrogenase $(\mathrm{LDH})$ release during reperfusion and infarct size, in this and the other series of experiments, was performed in all hearts subjected to ischaemia. Additional hearts ( $\mathrm{n}=9-10$ /group) of Control, Iso/Ade-10 and Iso/Ade-0 groups were freeze-clamped following 43 min preischaemia, ground under liquid nitrogen and stored at $-80^{\circ} \mathrm{C}$ for later analysis of PKC activity. Another set of Control, Iso/Ade- 0 and Iso/Ade Mix hearts ( $n=7-8$ in each group) were freeze-clamped at the end of the preishaemic protocol and used for the measurement of glycogen content.

In Series 2 experiments, four groups (6-9 hearts/group) were employed (Figure 1B). Two of the groups were Control and Iso/Ade with no washout period. In this series of experiments and in the Series 3 experiments, Iso was used at $10 \mathrm{nM}$. In preliminary experiments (data not shown), we found that this concentration of Iso might give even higher cardioprotective effect than $5 \mathrm{nM}$, which is important in terms of optimisation of the intervention, yet was still in the range of normal pharmacological dose. Another two groups of hearts were perfused with $0.2 \mu \mathrm{M}$ CsA for $8 \mathrm{~min}$ prior to ischaemia with or without the Iso/Ade treatment (Iso/Ade + CsA and CsA groups respectively).

Series 3 experiments were carried out on CAL and SO rats. Hearts were divided into four groups (5-6 hearts/ group): Control and Iso/Ade groups of CAL and $\mathrm{SO}$ rats. The experimental protocol of Control and Iso/Ade groups (Figure 2) in this series of experiments was similar to those in Series 2 (Figure 1B). Infarct size was not measured in these experiments because the CAL hearts had already developed an infarct as a result of the left descending coronary artery ligation.

Five additional CAL and 6 SO rats were used to confirm the development of heart failure. Body weight and tibia length were measured before the surgery and 16 weeks after the operation. After 16 weeks post-operation, the rats were killed by stunning and cervical dislocation, the hearts and lungs excised and the wet weight of the hearts, left ventricles and lungs were measured.

\section{Assays \\ PKC activity}

PKC activity was determined in freeze-clamped heart powders using a kit supplied by Promega according to the manufacturer's instructions. The assay relies on a change in charge of the fluorescent PepTag ${ }^{\odot} \mathrm{C} 1$ peptide from +1 to -1 following phosphorylation. Bands were visualized under UV light and the ratio of fluorescence intensity of phosphorylated to non-phosphorylated peptide was quantified using AlphaInotech ChemiImager 4400 with AlphaEase v5.5 software. 


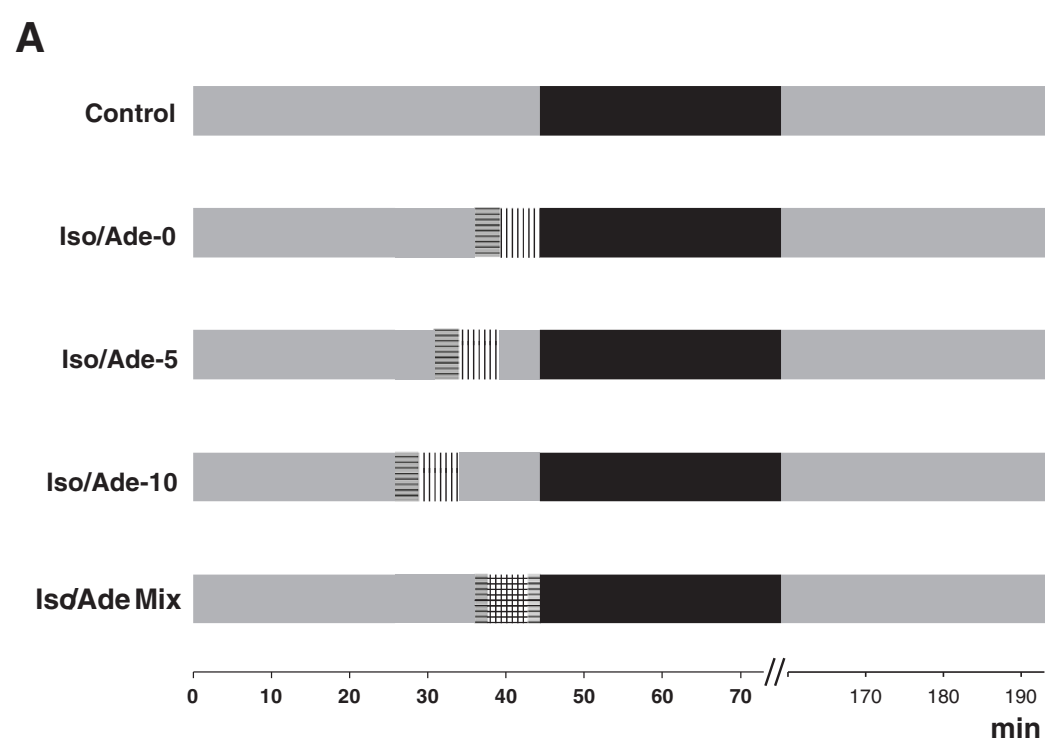

B

Control

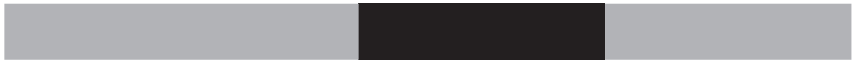

Iso/Ade

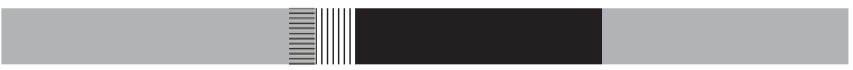

CsA
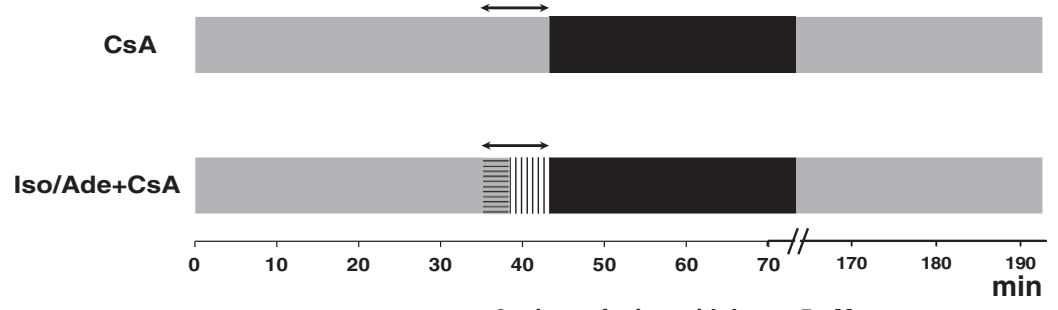

Krebs-Henseleit perfusion $\quad$ (A) or $10 \mathrm{nM}$ (B) in Iso/Ade

3 min perfusion with Iso at $5 \mathrm{nM}$

groups, $1.5 \mathrm{~min}$ in Iso/Ade Mix 㶾 5 min perfusion with 5 group (A).

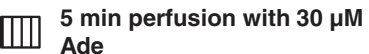

$\longleftrightarrow 8$ min perfusion with $0.2 \mu \mathrm{M}$ CsA

Global Normothermic

Ischaemia

Figure 1 Outline of the experimental protocols of series 1 and 2. Panel A - experimental protocol of the Series 1 . Iso/Ade-0, Iso/Ade-5 and Iso/Ade-10 - consecutive perfusion with isoproterenol and adenosine with 0, 5, and 10 min washout period respectively; Iso/Ade Mix perfusion with the mixture of isoproterenol and adenosine. Panel B - experimental protocol of the Series 2. Control; Iso/Ade - consecutive isoproterenol/ adenosine treatment with no washout period (similar to that of Iso/Ade-0 in the Series 1); CSA - perfusion with cyclosporine A; and Iso/Ade + CsA consecutive isoproterenol/adenosine treatment combined with perfusion with Cyclosporine A.

\section{Glycogen content in myocardium}

Glycogen content was assessed by measuring the glucose released from glycogen breakdown by amyloglucosidase based on the method described by Passonneau [16] and expressed as $\mu$ mol glucose per gram tissue. A sample $(70 \mathrm{mg})$ of the frozen tissue was suspended in $1 \mathrm{ml}$ of $0.3 \mathrm{M}$ perchloric acid $\left(4^{\circ} \mathrm{C}\right)$, homogenized with a Polytron and $50 \mu \mathrm{l}$ of the suspension were incubated with $0.5 \mathrm{ml}$ of $50 \mathrm{mM} \mathrm{Na}$ Acetate Buffer ( $\mathrm{pH}$ 5.5) containing
0.02\% BSA and $50 \mu \mathrm{g} / \mathrm{ml}$ amyloglucosidase (Sigma) at room temperature for $2 \mathrm{hr}$. The samples were then centrifuged at $10000 \mathrm{~g}$ for $10 \mathrm{~min}$ and glucose content was assessed using a Glucose (HK) Assay Kit (Sigma) according to the manufacturer's instruction. Briefly, $0.4 \mathrm{ml}$ of the supernatant was mixed with $1 \mathrm{ml}$ of the glucose assay reagent. Glucose content was measured spectrophotometrically $(340 \mathrm{~nm})$ after incubation at room temperature for $15 \mathrm{~min}$. 


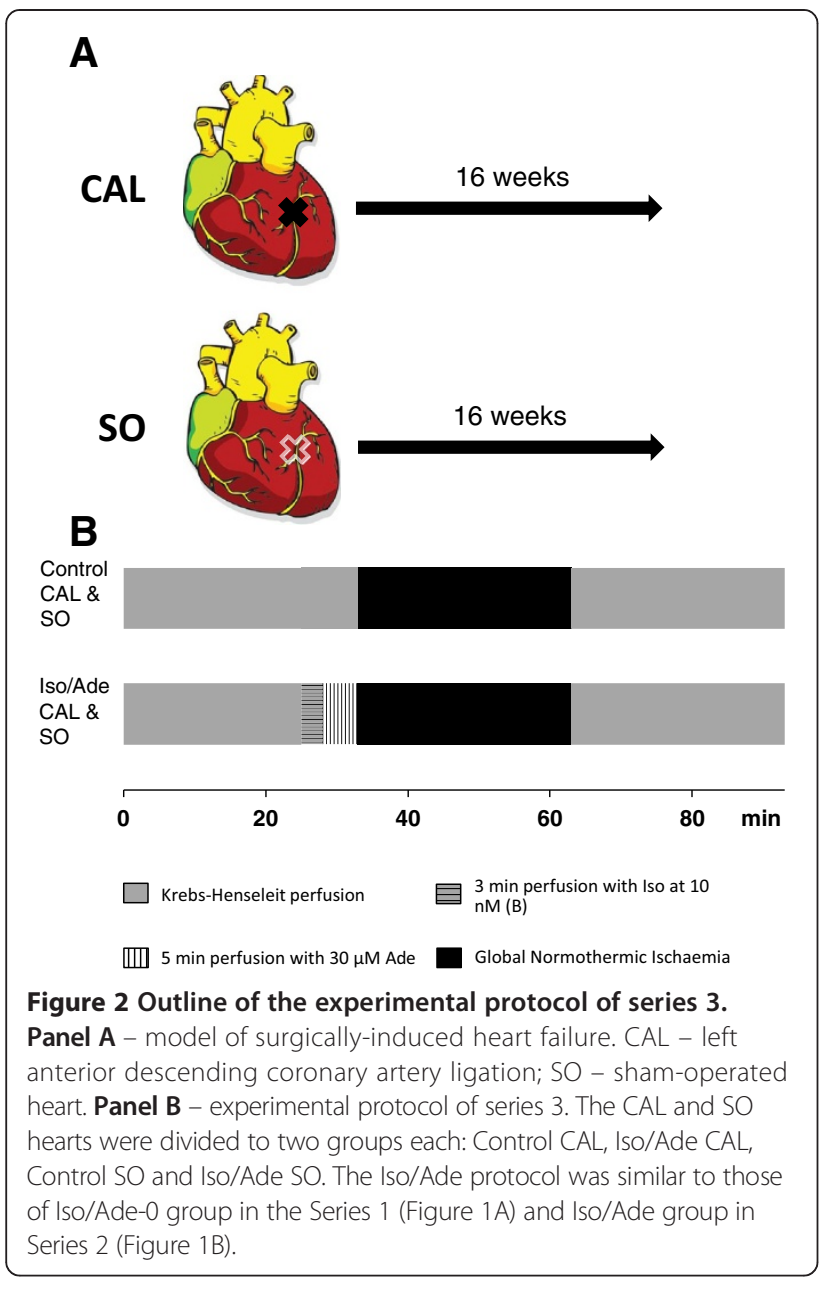

The quantity of glucose released from glycogen breakdown was determined by subtracting glucose in samples incubated without amyloglucosidase from those incubated with the enzyme.

\section{LDH activity}

LDH activity was determined in the effluent perfusate collected from the hearts of all groups prior to ischemia and during each 5 min over the first $30 \mathrm{~min}$ of reperfusion as described earlier [17] and modified at our lab: $80 \mu \mathrm{l}$ of the sample was added to $910 \mu \mathrm{l}$ of the buffer (pH 7.4) containing $100 \mathrm{mM}$ triethanolamine and $100 \mu \mathrm{M}$ reduced $\beta$-nicotinamide adenine dinucleotide (NADH). After addition of $10 \mu \mathrm{l}$ of $0.1 \mathrm{M}$ sodium pyruvate to the reaction mixture, the change of absorbance at $340 \mathrm{~nm}\left(\mathrm{~A}_{340}\right)$ was recorded using a spectrophotometer over $10 \mathrm{~min}$ at $37^{\circ} \mathrm{C}$. $\mathrm{LDH}$ activity was calculated according to the rate of $\mathrm{A}_{340}$ decrease.

\section{Infarct Size}

Infarct size was determined as described previously [18]. Hearts were stained with $1 \%$ of triphenyltetrazolium chloride after $2 \mathrm{~h}$ of reperfusion, frozen at $-20^{\circ} \mathrm{C}$ and sliced into 6 slices. Necrotic and intact areas of each side for each of heart slices were determined using AlphaEase v5.5 software and the total necrotic and intact area of ventricular myocardium of each heart was calculated. Since the entire heart was at risk from global ischemia, the infarct size was expressed dividing the sum of necrotic areas by the sum of total slice areas of the 6 slices to obtain the percentage of necrosis.

\section{Statistical Analysis}

Data are presented as mean \pm SEM. Statistical significances of the differences between groups were evaluated by one-way ANOVA followed by Tukey's multiple comparison post hoc test or two-tailed unpaired Student's $t$ test (morphometric measurements of CAL and SO rats in the Series 3 experiments) using the software GraphPad Prism, Version 4.03. Differences were considered significant where $\mathrm{P}<0.05$.

\section{Results}

Optimization of the protocol of Iso/Ade treatment with a clinically relevant dose of Isoproterenol

In the Iso/Ade group, RPP during heart perfusion with 5 $\mathrm{nM}$ Iso reached a maximum of $259.4 \pm 12.4 \%$ of the pretreatment value, mostly due to an increase in LVDP. RPP measured at the end of perfusion with $30 \mu \mathrm{M}$ Ade fell to $42.3 \pm 7.0 \%$ of the pre-treatment value, due to a significant reduction of both HR and LVDP. In Table 1 , we show that during reperfusion, recovery of RPP in the Control group was $28 \%$. However, perfusion with low Iso concentration $(5 \mathrm{nM})$ followed by $30 \mu \mathrm{M}$ Ade immediately prior to rendering the heart ischaemia (Iso/Ade-0 group), improved recovery in RPP to $76 \%$. Similar recovery was achieved when the duration of perfusion prior to commencing ischaemia was extended to 5 or $10 \mathrm{~min}$ (Iso/Ade-5 and Iso/Ade-10 groups).

The improved RPP recovery during reperfusion was accompanied by considerably reduced LVEDP and reflected improved recovery of LVDP with no significant change in HR. The time derivative during contraction was significantly higher in all three Iso/Ade groups than in Control group, whereas recovery of - $\mathrm{dP} / \mathrm{dt}$ was significantly improved only in hearts of the Iso/Ade-0 group. The parameters of haemodynamic function recovery did not differ significantly between the three Iso/Ade groups but were the highest in Iso/Ade- 0 group. Protection against necrotic damage showed a similar pattern to the recovery of haemodynamic function. Thus, LDH release during the first $30 \mathrm{~min}$ of reperfusion (Figure 3A) and infarct size at the end of reperfusion (Figure $3 \mathrm{~B}$ ) were significantly lower in Iso/Ade groups compared to Control. However infarct size in the group Iso/Ade-0 was notably lower compared to both Control and Iso/Ade-5 groups. 
Table 1 Haemodynamic function in Iso/Ade groups of hearts with different duration of the washout period

\begin{tabular}{|c|c|c|c|c|c|c|}
\hline \multirow[t]{2}{*}{ Parameters } & \multirow{2}{*}{$\begin{array}{c}\text { Preiscaemic } \\
\text { equilibration } \\
(n=34)\end{array}$} & \multicolumn{5}{|c|}{ Reperfusion (Percentage preischaemic value) } \\
\hline & & \multicolumn{2}{|c|}{ Control $(n=7)$} & \multirow{2}{*}{$\begin{array}{c}\text { Iso/Ade-0 }(\mathbf{n}=\mathbf{1 0}) \\
88.9 \pm 7.2^{* * *}\end{array}$} & \multirow{2}{*}{$\begin{array}{c}\text { Iso/Ade-5 }(\mathbf{n}=\mathbf{9}) \\
73.6 \pm 8.3^{*}\end{array}$} & \multirow{2}{*}{$\begin{array}{c}\text { Iso/Ade-10 }(\mathbf{n}=\mathbf{8}) \\
78.3 \pm 14.9^{*}\end{array}$} \\
\hline LVDP (mmHg) & $75.1 \pm 2.5$ & & $29.4 \pm 5.7$ & & & \\
\hline HR (beat/min) & $277.6 \pm 4.2$ & & $102.5 \pm 11.4$ & $86.9 \pm 5.6$ & $94.5 \pm 3.2$ & $71.6 \pm 8.9$ \\
\hline $\mathrm{RPP}(\mathrm{mmHg} \cdot$ beat/min $)$ & $20802 \pm 713$ & $\%$ of initial values & $28.5 \pm 5.2$ & $76.3 \pm 6.9 * * *$ & $68.5 \pm 7.0 * *$ & $73.3 \pm 10.5 * *$ \\
\hline$+\mathrm{dP} / \mathrm{dt}(\mathrm{mmHg} / \mathrm{s})$ & $3082 \pm 117$ & & $30.2 \pm 2.5$ & $65.2 \pm 6.9 * *$ & $58.6 \pm 5.3 *$ & $60.4 \pm 8.5 *$ \\
\hline$-\mathrm{dP} / \mathrm{dt}(\mathrm{mmHg} / \mathrm{s})$ & $-2027 \pm 83$ & & $42.7 \pm 4.4$ & $85.5 \pm 7.2 * *$ & $65.6 \pm 8.2$ & $71.5 \pm 8.9$ \\
\hline Maximal LVEDP at Reperfusion $(\mathrm{mmHg})$ & - & $98.7 \pm 7$ & & $50.0 \pm 4.4^{* * *}$ & $62.1 \pm 8.2 * *$ & $63.2 \pm 6.5 * *$ \\
\hline
\end{tabular}

Haemodynamic function was determined at the end of the equilibration period prior to ischaemia and after 60 min reperfusion.

${ }^{*} \mathrm{P}<0.05,{ }^{* *} \mathrm{P}<0.01,{ }^{* * *} \mathrm{P}<0.001$ vs. Control.

PKC activity in Iso/Ade-0 group, measured at the end of the pre-ischaemic protocol, was 1.5 fold higher compared to both Control and Iso/Ade-10 groups (Figure 4).

Glycogen content was halved in hearts treated consecutively with Iso and Ade, but simultaneous perfusion of hearts with these drugs had no significant effect on this parameter (Figure 5).

\section{Effect of the combined treatment with Iso, Ade and cyclosporine $\mathrm{A}$ on ischaemia/reperfusion injury}

In the Iso/Ade group of the second series of experiments, $\mathrm{RPP}$ during heart perfusion with $10 \mathrm{nM}$ of Iso reached a maximum of $346.1 \pm 26.9 \%$ of the pre-treatment value, significantly exceeding RPP increase in Series 1 hearts treated with $5 \mathrm{nM}$ Iso $(\mathrm{P}<0.05)$. This elevation of $\mathrm{RPP}$ was mostly due to increase in LVDP. RPP measured at the end of perfusion with $30 \mu \mathrm{M}$ Ade fell to $36.8 \pm 7.6 \%$ of the pre-treatment value due to a significant reduction of both HR and LVDP. The MPTP inhibitor CsA significantly increased RPP recovery compared to Control (Table 2) and was as effective as the consecutive Iso/Ade treatment in terms of reduction of infarct size (Figure 6B). LDH activity in the effluent perfusate during reperfusion was also significantly reduced in these hearts (Figure 6A). However perfusion of hearts with CsA together with the consecutive Iso/Ade treatment (Iso/Ade + CsA group), at clinically relevant concentration of these drugs, did not bring about any additive protective effect; infarct size and $\mathrm{LDH}$ release were similar to those of Iso/Ade group.

\section{Consecutive Iso/Ade treatment protects hearts with surgically-induced heart failure}

At the time of the ligation of left coronary artery, no significant difference in body weight or tibia length was detected between sham-operated (SO) and Coronary Artery Ligation (CAL) animals. After 16 weeks post-surgery, there was no difference in body weight between the two groups. However, significant increases in heart and lung weights, heart and lung to body weight ratios, as well as tibia length to body weight ratio in the CAL animals were observed
(Table 3) indicating both cardiac hypertrophy and cardiac insufficiency.

During heart perfusion with $10 \mathrm{nM}$ of Iso, RPP reached a maximum of $401.3 \pm 30.8 \%$ of the pre-treatment value in SO group and $343.3 \pm 52.4 \%$ in CAL group, mostly due to increase in LVDP. This parameter in the SO group, but not in the CAL group, was significantly higher than in Series 1 where the hearts were treated with $5 \mathrm{nM}$ Iso $(\mathrm{P}<0.01)$. RPP measured at the end of perfusion with $30 \mu \mathrm{M}$ Ade fell to $43.5 \pm 8.0 \%$ of the pre-treatment value in $\mathrm{SO}$ hearts and to $56.6 \pm 9.3 \%$ in CAL hearts, due to a significant reduction of $H R$ and return of LVDP to the pre-treatment value. There was no significant difference in changes of RPP in response to the perfusion with Ade between SO and CAL groups. Haemodynamic function recovery of both SO and CAL groups of hearts following ex-vivo ischaemia/reperfusion was very poor and did not significantly differ from each other (Table 4). Thus, LVDP and RPP reached only $20-25 \%$ of the initial preischaemic values. Iso/Ade treatment significantly improved haemodynamic function recovery of both SO and CAL hearts with RPP reaching $70 \%$ of the preischaemic value. $\mathrm{LDH}$ activity during reperfusion was highest in Control CAL hearts and was significantly higher not only than Iso/Ade treated hearts of SO and CAL groups but also than Control SO hearts (Figure 7). Thus, necrotic damage was greatest in the untreated failing hearts but Iso/Ade treatment was able to protect the failing hearts at least as effectively as the shamoperated ones.

\section{Discussion}

Optimisation of the Iso/Ade treatment and its translational significance

Our results show that Iso at its low dose (5-10 nM), followed by the perfusion with $30 \mu \mathrm{M}$ Ade, can successfully protect hearts against ischaemia/reperfusion injury. In fact, when Iso was perfused at $10 \mathrm{nM}$, the protective effect was similar to the higher concentration of Iso (200 $\mathrm{nM}$ ) used in our previous experiments [4]. Since both Iso and Ade are used in clinical settings and can protect 

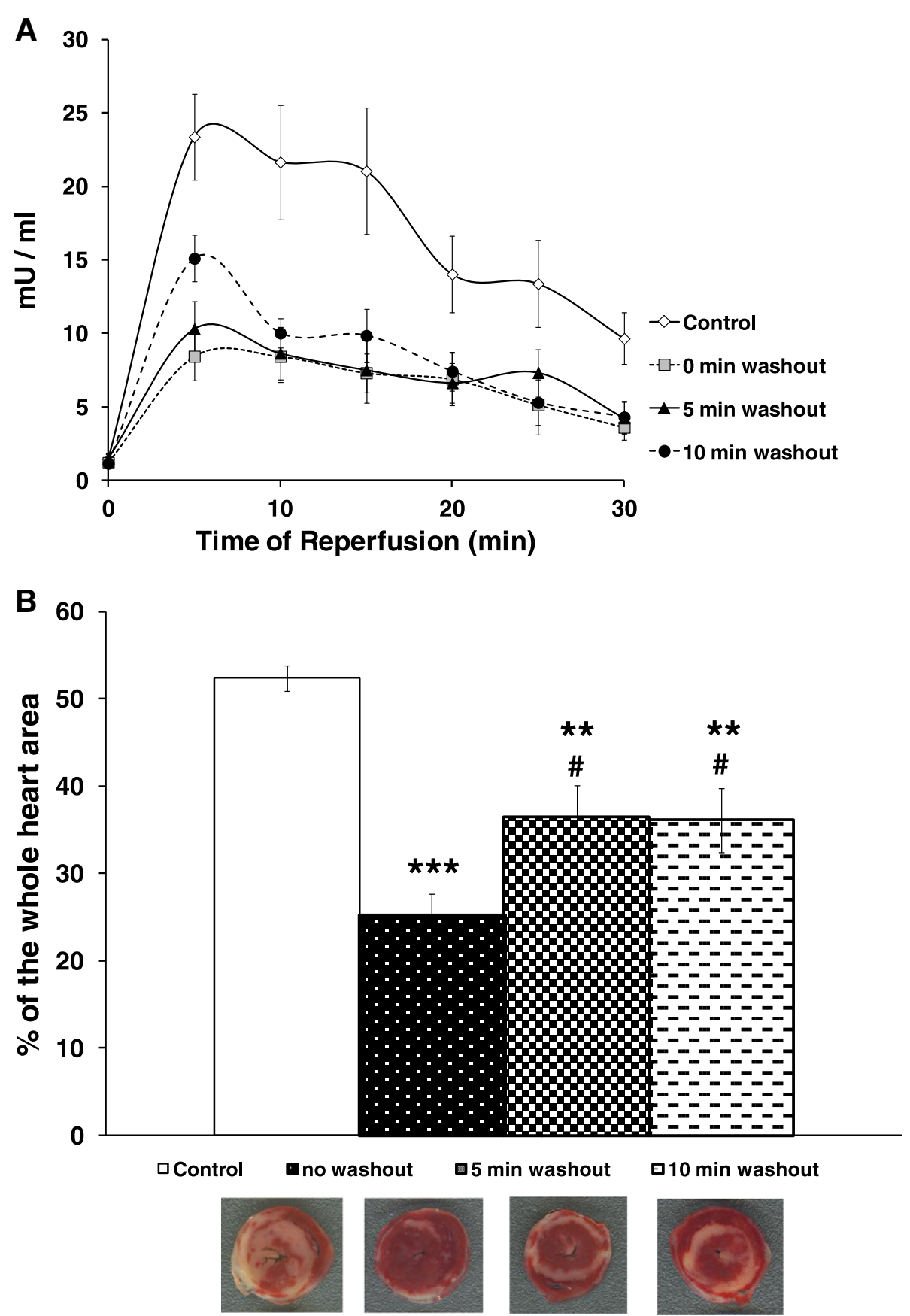

Figure 3 Lactate dehydrogenase (LDH) activity and infarct size in hearts of the Series 1 . The experiments were carried out on Control $(n=7)$ and Iso/Ade groups with $0(n=9), 5(n=9)$, and $10(n=9)$ min washout periods. Panel A - LDH activity in the effluent perfusate during 30 min reperfusion. Differences between Control and all three Iso/Ade groups (measured for the corresponding data points) were statistically significant $(\mathrm{P}<0.05)$ at all data points. There was no significant difference in LDH activity between the Iso/Ade groups. Panel B - Infarct size. Mean \pm SEM (columns) and representative images of the corresponding heart slices stained with 2,3,5-triphenyltetrazolium chloride (TTC). ** $\mathrm{P}<0.01,{ }^{* * *} \mathrm{P}<0.001$ vs. Control; \# $\mathrm{P}<0.05$ vs. Iso/Ade group with zero min washout period.

hearts at clinically relevant doses, the consecutive Iso/ Ade treatment has strong potential for translation into clinical practice. Furthermore, the use of these drugs at low doses will minimise other drug interactions. This work also demonstrates that there is a window of at least 10 min within which the index ischaemia can be started and strong cardioprotection still be observed. However, it is clear that, the shorter the period between the end of 

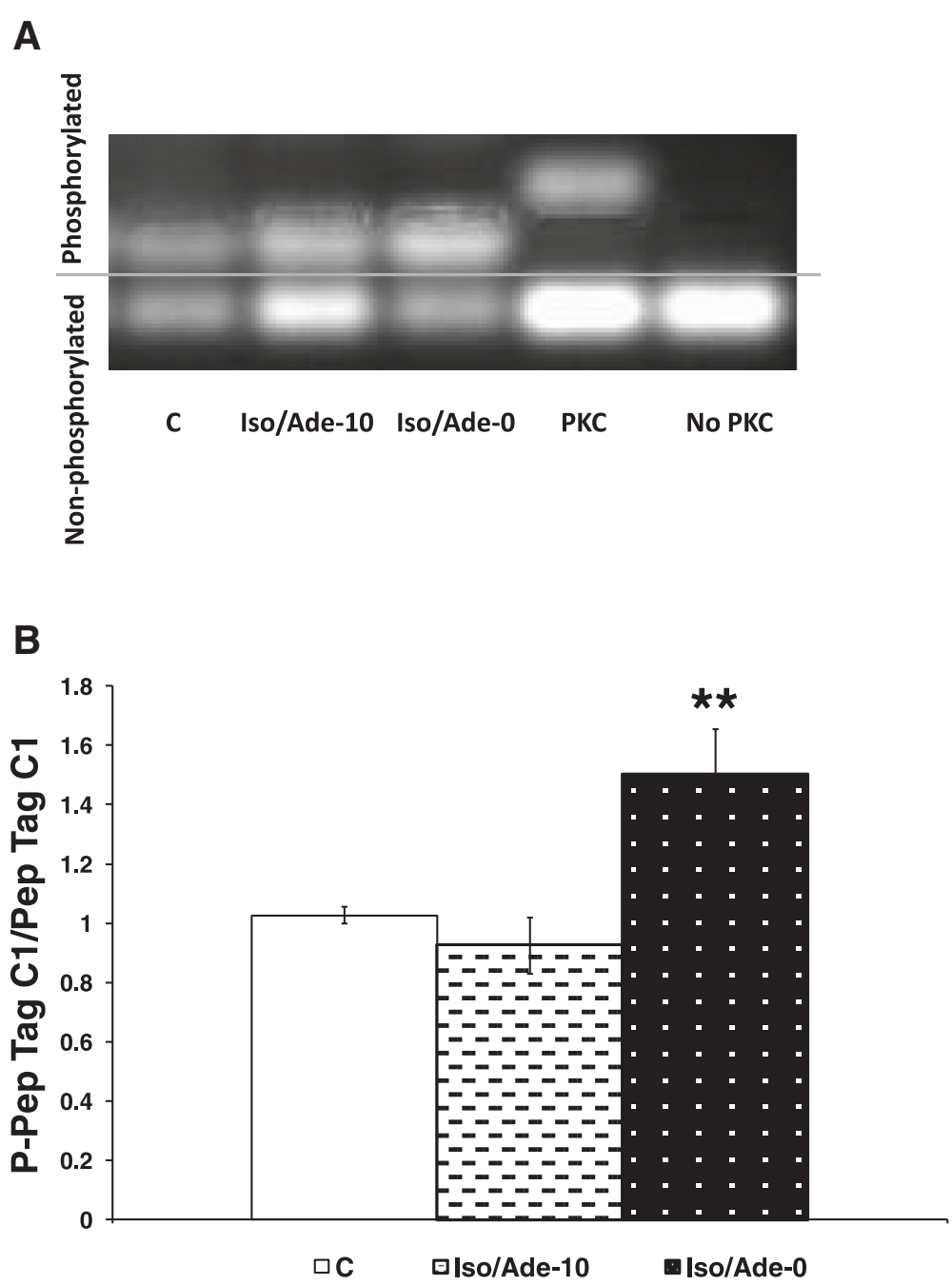

Figure 4 Protein Kinase C (PKC) activity in hearts of Experimental Series 1. PKC activity was measured using non-radioactive PepTag ${ }^{\oplus}$ assay and is expressed as Mean \pm SEM of a ratio of fluorescence intensity of phosphorylated and non-phosphorylated PepTag ${ }^{\circledR}$ C1 peptide (Panel B) which is proportional to the PKC activity. PKC activity was measured in Control $(C, n=10)$, Iso/Ade-0 $(n=9)$ and Iso/Ade-10 $(n=9)$ groups. Panel A - Representative image of the phosphorylated and non-phosphorylated PepTag ${ }^{\oplus}$ C1 peptide under UV light. ${ }^{* *} \mathrm{P}<0.01$ vs. Control.

the treatment and the start of ischaemia the higher the cardioprotective efficacy of the treatment will be. Thus in our experiments, infarct size in hearts with no washout period (Iso/Ade-0 group) was significantly smaller than in both Control and Iso/Ade-5 hearts. Furthermore, only in Iso/Ade- 0 hearts was $-\mathrm{dP} / \mathrm{dt}$ recovery significantly higher than in controls. This might be explained by the index ischaemia in these hearts commencing while PKC remained activated, potentially leading to more effective survival signalling.

Having established that clinically-relevant doses of isoproteronol and adenosine are cardioprotective and was optimal when applied immediately prior to ischaemia, the next step was to identify the target of this powerful cardioprotective intervention. Our earlier work suggested that reduced ROS production and consequent inhibition of MPTP opening were likely targets [4]. If this is the case, protection should not be further enhanced by direct inhibition of the MPTP by Cyclosporin A and we confirmed this to be the case.

\section{Role of MPTP and Cardiac Glycogen in cardioprotection by Iso/Ade treatment}

We [9] and others [19-21] have shown that MPTP opening is a key factor of irreversible damage during reperfusion and that its prevention underlies cardioprotection by a variety of preconditioning [12] and post-conditioning protocols [10]. Thus, it was hypothesized that the protection associated with ischaemic preconditioning could be attributed to a decreased probability of MPTP opening $[9,22]$. Ovize et al. proposed MPTP inhibition as the effector of ischaemic postconditioning [10]. It has been 


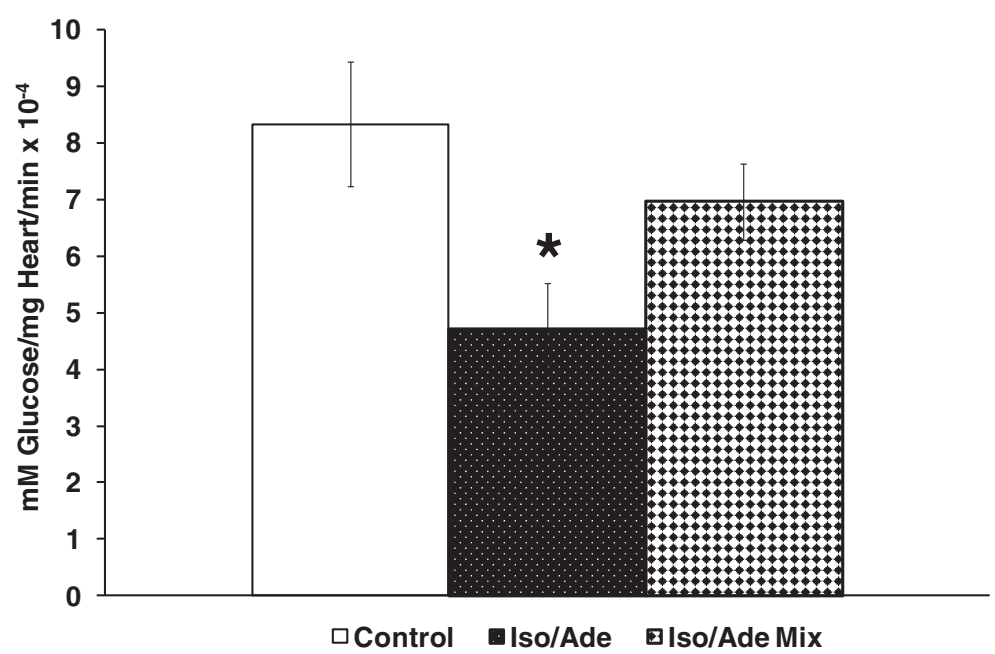

Figure 5 Glycogen content in hearts of Experimental Series 1. Glycogen content in myocardium of Control hearts $(n=8)$, hearts treated consecutively with Iso and Ade (Iso/Ade group, $\mathrm{n}=8$ ) and hearts treated with the mixture of Iso and Ade (Iso/Ade Mix, $\mathrm{n}=7$ ) was measured prior to ischaemia. ${ }^{*} P<0.05$ vs. control.

shown that the protective effect of remote ischaemic preconditioning [11] and a variety of pharmacologicallyinduced cardioprotective interventions [12] also rely on the MPTP inhibition. In our recent study with Iso/Ade treatment, where Iso was used at relatively high concentration $(200 \mathrm{nM})$, the treatment resulted in a dramatic reduction of calcium-induced mitochondria swelling implicating MPTP inhibition as an end effector of the cardioprotective mechanism [4].

In the present work, we used the MPTP inhibitor CsA in order to confirm a role for MPTP inhibition in the cardioprotection afforded by Iso/Ade treatment. We found that CsA improved RPP recovery and significantly reduced necrotic damage to myocardium during reperfusion. These results are consistent with earlier data shown by us [23,24] and others [25-27] confirming a cardioprotective effect of CsA. However, no additive cardioprotective effect was seen when consecutive treatment with $10 \mathrm{nM}$ isoproterenol and $30 \mu \mathrm{M}$ adenosine was combined with CsA treatment.
These data suggest that cardioprotection by the Iso/Ade treatment is mediated through inhibition of the MPTP.

It has been reported that protection via ischaemic preconditioning is associated with glycogen depletion leading to less anaerobic glycolysis during the subsequent prolonged ischaemia, and hence reduced accumulation of lactate and $\mathrm{H}^{+}$. This causes a smaller decrease in intracellular $\mathrm{pH}$ during ischaemia and thus less compensatory increases in intracellular $\mathrm{Na}^{+}$and $\mathrm{Ca}^{2+}$ [28]. The decreased $\mathrm{Ca}^{2+}$ loading will reduce the likelihood that the MPTP opens which might partially explain the observed cardioprotection [29]. Glycogen breakdown has also been linked to attenuation of the loss of hexokinase 2 (HK-2) binding to mitochondria that occurs during ischaemia [30]. The binding of HK-2 plays an antiapoptotic role and inhibits MPTP opening [31,32] and there is an inverse correlation between the extent of HK-2 binding at the end of ischaemia and infarct size [30]. Increase HK-2 binding at the end of ischaemia

Table 2 Haemodynamic function in hearts of Control and Iso/Ade groups with or without cyclosporine A

\begin{tabular}{|c|c|c|c|c|c|c|}
\hline \multirow[t]{2}{*}{ Parameters } & \multirow{2}{*}{$\begin{array}{c}\text { Pre- } \\
\text { Ischaemia } \\
(\mathrm{n}=24)\end{array}$} & \multicolumn{5}{|c|}{ Reperfusion (Percentage preischaemic value) } \\
\hline & & Control (n & = 7) & Iso/Ade $(n=6)$ & $\operatorname{CsA}(n=5)$ & Iso/Ade + CsA $(n=6)$ \\
\hline LVDP (mmHg) & $75.8 \pm 2.9$ & & $39.0 \pm 6.6$ & $100.5 \pm 14.2 *$ & $89.5 \pm 14.4$ & $97.8 \pm 16.9 *$ \\
\hline HR (beat/min) & $288.3 \pm 3.9$ & & $96.0 \pm 3.5$ & $93.1 \pm 3.1$ & $99.3 \pm 4.7$ & $102.9 \pm 2.9$ \\
\hline $\mathrm{RPP}(\mathrm{mmHg} \cdot \mathrm{beat} / \mathrm{min})$ & $21854 \pm 889$ & $\%$ of initial values & $38.8 \pm 7.5$ & $93.0 \pm 12.0 * *$ & $86.3 \pm 13.3 *$ & $100.3 \pm 11.2^{* *}$ \\
\hline$+\mathrm{dP} / \mathrm{dt}(\mathrm{mmHg} / \mathrm{s})$ & $2969 \pm 94$ & & $34.2 \pm 4.6$ & $81.3 \pm 12.3 *$ & $66.4 \pm 13.3$ & $87.2 \pm 14.3 *$ \\
\hline$-\mathrm{dP} / \mathrm{dt}(\mathrm{mmHg} / \mathrm{s})$ & $-2074 \pm 84$ & & $42.7 \pm 4.7$ & $93.6 \pm 12.4^{* *}$ & $71.7 \pm 8.3$ & $90.8 \pm 13.5 *$ \\
\hline Maximal LVEDP at Reperfusion (mmHg) & - & $98.6 \pm 5$ & & $53.9 \pm 13.2^{* *}$ & $78.2 \pm 8.6$ & $65.9 \pm 4.2 *$ \\
\hline
\end{tabular}

Haemodynamic function was determined at the end of equilibration period prior to ischaemia and after 60 min reperfusion. The perfusion protocol of Iso/Ade group in this series of experiments was similar to that of Iso/Ade-0 group in Experimental Series 1.

* $\mathrm{P}<0.05$, ** $\mathrm{P}<0.01,{ }^{* * *} \mathrm{P}<0.001$ vs. Control. 



Figure 6 LDH activity and infarct size in hearts in Experimental Series 2. The experiments were carried out on Control $(n=9)$, Iso/Ade $(n=9)$, cyclosporine $A(C s A, n=6)$ and Iso/Ade + CsA $(n=8)$ groups of hearts. The perfusion protocol of Iso/Ade group in this series of experiments was similar to that of Iso/Ade-0 group in Experimental Series 1. Panel A - LDH activity in the effluent perfusate during 30 min reperfusion. Differences between control and three other groups (measured for the corresponding data points) were statistically significant $(P<0.05)$ at all data points. There was no significant difference in LDH activity between the three intervention groups. Panel B - Infarct size. Mean \pm SEM (columns) and representative images of the corresponding heart slices stained with $\Pi T C$. ${ }^{* *} \mathrm{P}<0.01,{ }^{* * *} \mathrm{P}<0.001$ vs. Control.

can be mediated, at least in part, by decreasing tissue glycogen content prior to ischaemia. This leads to a reduction in tissue glucose-6-phosphate levels and acidification during ischaemia, which mediate HK-2 dissociation from mitochondria [30]. The data of Figure 5 confirm that the consecutive Iso/Ade treatment also depleted glycogen content considerably consistent with this mechanism, whereas the reduction in glycogen content following simultaneous application of the drugs was not significant and was accompanied by considerably less cardioprotection. However, reduced glycogen content cannot entirely account for the cardioprotection since we have

Table 3 Effects of the left coronary artery ligation (CAL) on cardiac morphology

\begin{tabular}{|c|c|c|c|c|c|c|c|c|}
\hline Procedure & BW (g) & $\mathrm{HW}(\mathrm{g})$ & LW (g) & Tibia (mm) & $\mathrm{HW} / \mathrm{BW}(\mathrm{mg} / \mathrm{g})$ & LW/BW (mg/g) & HW/Tibia (mg/mm) & LW/Tibia (mg/mm) \\
\hline Sham $(n=6)$ & $502.2 \pm 8.0$ & $1.56 \pm 0.04$ & $1.62 \pm 0.04$ & $46.7 \pm 0.5$ & $3.1 \pm 0.1$ & $3.2 \pm 0.1$ & $3.3 \pm 0.1$ & $3.5 \pm 0.1$ \\
\hline CAL $(n=5)$ & $491.4 \pm 25.6$ & $2.42 \pm 0.20 * *$ & $1.97 \pm 0.13 *$ & $46.1 \pm 0.6$ & $4.9 \pm 0.4^{* * *}$ & $4.0 \pm 0.1 * * *$ & $5.3 \pm 0.4^{* * *}$ & $4.3 \pm 0.2^{* *}$ \\
\hline
\end{tabular}

BW - body weight; HW - heart weight; LW - lung weight. ${ }^{*} \mathrm{P}<0.05,{ }^{* *} \mathrm{P}<0.01,{ }^{* * *} \mathrm{P}<0.001$ vs. Sham. 
Table 4 Haemodynamic function in coronary artery ligation (CAL) and sham-operated (SO) Iso/Ade groups of hearts

\begin{tabular}{|c|c|c|c|c|c|c|}
\hline \multirow[t]{2}{*}{ Parameters } & \multirow{2}{*}{$\begin{array}{l}\text { Pre-Ischaemia } \\
\qquad(n=20)\end{array}$} & \multicolumn{5}{|c|}{ Reperfusion (Percentage preischaemic value) } \\
\hline & & \multicolumn{2}{|c|}{ Control SO $(n=5)$} & Iso/Ade SO $(n=5)$ & Control CAL $(n=5)$ & Iso/Ade CAL $(n=5)$ \\
\hline LVDP (mmHg) & $75.2 \pm 4.3$ & \multirow{5}{*}{$\%$ of initial values } & $24.1 \pm 3.9$ & $65.4 \pm 9.1 *$ & $20.7 \pm 4.0$ & $69.6 \pm 15.2^{* *}$ \\
\hline HR (beat/min) & $271.1 \pm 7.8$ & & $89.1 \pm 19.4$ & $107.5 \pm 7.6$ & $94.4 \pm 3.0$ & $99.0 \pm 4.5$ \\
\hline $\mathrm{RPP}(\mathrm{mmHg} \cdot$ beat$/ \mathrm{min})$ & $20454 \pm 1333$ & & $22.5 \pm 5.9$ & $66.3 \pm 9.8 *$ & $19.7 \pm 4.0$ & $69.0 \pm 16.3 *$ \\
\hline$+\mathrm{dP} / \mathrm{dt}(\mathrm{mmHg} / \mathrm{s})$ & $2596 \pm 154$ & & $31.7 \pm 3.3$ & $82.4 \pm 20.8$ & $66.3 \pm 15.8$ & $87.2 \pm 14.3$ \\
\hline$-\mathrm{dP} / \mathrm{dt}(\mathrm{mmHg} / \mathrm{s})$ & $-1868 \pm 138$ & & $37.8 \pm 2.7$ & $86.5 \pm 22.1 * *$ & $40.4 \pm 4.5$ & $77.0 \pm 14.3$ \\
\hline $\begin{array}{l}\text { Maximal LVEDP at } \\
\text { Reperfusion }(\mathrm{mmHg})\end{array}$ & - & \multicolumn{2}{|c|}{$91.9 \pm 19.1$} & $28.7 \pm 12.2 *$ & $67.3 \pm 9.9$ & $20.2 \pm 21.1$ \\
\hline
\end{tabular}

Haemodynamic function was determined at the end of equilibration period prior to ischaemia and after 60 min reperfusion. The perfusion protocol of Iso/Ade groups in this series of experiments was similar to that of Iso/Ade-0 group in Experimental Series 1.

${ }^{*} \mathrm{P}<0.05$, ${ }^{* *} \mathrm{P}<0.01$ vs. a corresponding Control.

shown previously that a similar decrease in glycogen could be induced by high dose of isoproterenol alone, yet this treatment was less cardioprotective than the consecutive treatment with isoproterenol and adenosine [4]. Thus adenosine may contribute to cardioprotection through glycogen independent mechanisms that improve haemodynamic function recovery during reperfusion and inhibit MPTP opening [4].

\section{Consecutive Iso/Ade treatment protects hearts with surgically-induced heart failure}

Since the majority of patients undergoing cardiac surgery suffer from co-morbid illnesses, for clinical use of consecutive Iso/Ade treatment it is necessary to confirm its efficacy in an experimental model of the diseased heart $[33,34]$. In general, the presence of co-morbid illness renders the myocardium resistant to cardioprotection against infarction by physical or pharmacological stimuli [14]. It has been found that compensated left

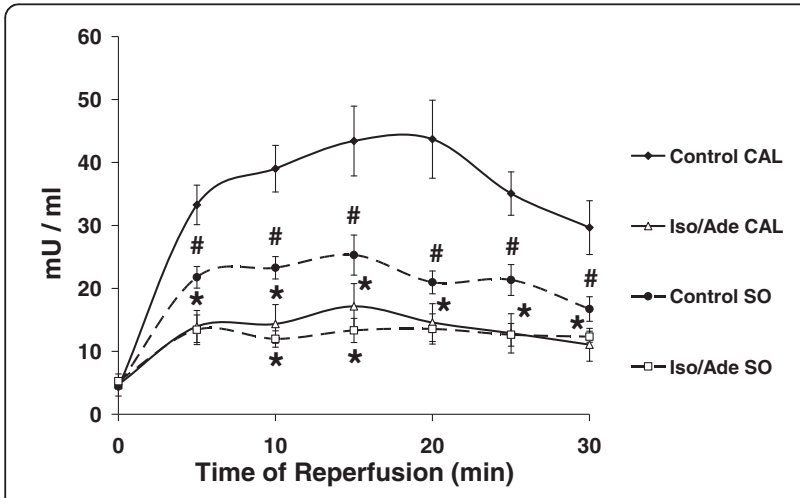

Figure 7 LDH activity in hearts of Experimental Series 3. LDH activity was measured in the effluent perfusate during $30 \mathrm{~min}$ reperfusion. The perfusion protocol of Iso/Ade groups in this series of experiments was similar to that of Iso/Ade-0 group in the Experimental Series 1. LDH activity is presented for Control and Iso/Ade groups in sham-operated hearts ( $\mathrm{SO}, \mathrm{n}=6$ in each group) and failing hearts after the left descending coronary artery ligation (CAL, $n=5$ in control and $\mathrm{n}=6$ in Iso/Ade group). ${ }^{*} \mathrm{P}<0.05$ vs. corresponding Control (either $\mathrm{SO}$ or $\mathrm{CAL}$ ); \# $\mathrm{P}<0.05$ vs. Control CAL. ventricular hypertrophy does not compromise the efficacy of cardioprotective interventions such as ischaemic preconditioning but heart failure abolishes any beneficial effect $[33,35,36]$. Heart failure is a complex clinical syndrome, the etiology of which generally stems from preexisting ischaemic heart/coronary artery disease, or it is of non-ischaemic and/or idiopathic origin [37,38]. It is characterized by the progressive inability of the heart to fill with, and eject adequate amounts of blood to meet the needs of the body [39].

Ligation of the left descending coronary artery in our experiments rendered a significant increase in heart and lung weight to body weight and tibia length ratios (Table 3) in 16 weeks after the surgery indicating both cardiac hypertrophy and cardiac insufficiency.

Consistent with the dose-dependent effect, preischaemic perfusion of SO hearts with $10 \mathrm{nM}$ Iso resulted in a significantly higher increase of RPP than in hearts of Series 1 treated with $5 \mathrm{nM}$ Iso. In the CAL group, RPP was also increased considerably during perfusion with $10 \mathrm{nM}$ Iso but this was not significantly higher than in hearts treated with $5 \mathrm{nM}$ Iso. Thus, although the myocardium of rats with surgically-induced heart failure model showed somewhat reduced $\beta$-adrenergic reactivity, they still responded well to the $\beta$-adrenergic stimulation. Control CAL hearts suffered more severe necrotic damage during reperfusion than their sham-operated counterparts as reflected in the released LDH activity during reperfusion which was twice as high in these hearts as compared to the non-treated SO hearts (Figure 7). Nevertheless, the haemodynamic function recovery in Iso/Ade treated CAL hearts was similar to SO hearts whilst LDH release was suppressed more strongly than in the sham-operated hearts. This implies that heart failure in our experiments did not compromise the cardioprotective effect of consecutive Iso/Ade treatment and that the survival signal transduction pathways activated by this treatment were still responsive in spite of the developed heart failure.

The results of our study may represent a significant step towards translation of the consecutive Iso/Ade treatment 
to cardiac surgery involving ischaemic cardioplegic arrest and cardiopulmonary bypass.

\section{Conclusions}

The novel findings of our study are as follows:

1. Strong cardioprotective effect of PKA/PKC activation can be achieved by heart perfusion with Iso at the clinically relevant concentration of $5-10$ $\mathrm{nM}$ followed by perfusion with Ade.

2. The cardioprotective efficacy of PKA/PKC activation remains for at least $10 \mathrm{~min}$ after the intervention. From a practical point of view, this means that this protective intervention will still be effective even if it is performed some time before the ischaemic incident (i.e. cardiac surgery with cardioplegic arrest).

3. In contrast to a number of studies showing loss or attenuation of cardioprotection in diseased or ageing hearts, consecutive Iso/Ado treatment can protect the failing heart effectively. This efficacy of the treatment in a relevant comorbidity model increases the likelihood of successful translation into clinical practice.

4. Our data suggest a key role of MPTP inhibition in the cardioprotective effect of this treatment. Glycogen depletion of the myocardium induced by the treatment prior to ischaemia is implicated in the mechanism underlying MPTP inhibition and cardioprotection.

\footnotetext{
Abbreviations

Ade: Adenosine; CAL: Coronary artery ligated; CsA: Cyclosporine A; Iso: Isoproterenol; KH: Krebs-Henseleit buffer; LDH: Lactate dehydrogenase; LVDP: Left ventricular developed pressure; LVEDP: Left ventricular end-diastolic pressure; LVSP: Left ventricular systolic pressure; MPTP: Mitochondria permeability transition pore; PKA: Protein kinase A; PKC: Protein kinase C; SO: Sham-operated; RPP: Rate-pressure product; TP: Temperature preconditioning; TTC: 2,3,5-triphenyltetrazolium chloride; +dP/dt: Time derivatives of pressure during contraction; - dP/dt: Time derivative of pressure during relaxation.
}

\section{Competing interests}

The authors declare that they have no competing interests.

\begin{abstract}
Authors' contributions
IK participated in design of the study, carried out data acquisition, data analysis and drafted the manuscript. APH participated in design of the study and helped to draft the manuscript. SMB provided the surgically-induced model of heart failure. DJD took part in the data acquisition and data analysis. AFJ participated in design and coordination of the study and helped to draft the manuscript. MSS conceived the study, participated in its design and coordination and helped to draft the manuscript. All authors have read and approved the final manuscript.
\end{abstract}

\section{Acknowledgements}

The authors thank Clive Orchard (Bristol) for his contribution to the establishment of the heart failure model. This work was supported by a grant from the British Heart Foundation (PG/11/79/29125). Simon M Bryant was supported by a British Heart Foundation project grant (PG/10/91/28644).
Received: 17 February 2014 Accepted: 13 May 2014

Published: 21 May 2014

\section{References}

1. Depre C, Taegtmeyer H: Metabolic aspects of programmed cell survival and cell death in the heart. Cardiovasc Res 2000, 45:538-548.

2. Eltzschig HK, Eckle T: Ischemia and reperfusion-from mechanism to translation. Nat Med 2011, 17:1391-1401.

3. Khaliulin I, Clarke SJ, Lin H, Parker JE, Suleiman MS, Halestrap AP: Temperature preconditioning of isolated rat hearts - a potent cardioprotective mechanism involving a reduction in oxidative stress and inhibition of the mitochondrial permeability transition pore. J Physio/ 2007, 581:1147-1161.

4. Khaliulin I, Parker JE, Halestrap AP: Consecutive pharmacological activation of PKA and PKC mimics the potent cardioprotection of temperature preconditioning. Cardiovasc Res 2010, 88:324-333.

5. Khaliulin I, Halestrap AP, Suleiman MS: Temperature preconditioning is optimal at $26^{\circ} \mathrm{C}$ and confers additional protection to hypothermic cardioplegic ischemic arrest. Exp Biol Med 2011, 236:736-745.

6. Gilmore K, Nanyanzi C: Pharmacology of vasopressors and inotropes. Update Aneasthesia 1999, 10:1-2.

7. Mentzer RM Jr, Birjiniuk V, Khuri S, Lowe JE, Rahko PS, Weisel RD, Wellons HA, Barker ML, Lasley RD: Adenosine myocardial protection: preliminary results of a phase II clinical trial. Ann Surg 1999, 229:643-649. discussion 649-650.

8. Liu R, Xing J, Miao N, Li W, Liu W, Lai Y-Q, Luo Y, Ji B: The myocardia protective effect of adenosine as an adjunct to intermittent blood cardioplegia during open heart surgery. Eur J Cardiothorac Surg 2009, 36:1018-1023.

9. Halestrap AP, Clarke SJ, Khaliulin I: The role of mitochondria in protection of the heart by preconditioning. Biochim Biophys Acta 2007, 1767:1007-1031.

10. Argaud L, Gateau-Roesch O, Raisky O, Loufouat J, Robert D, Ovize M: Postconditioning inhibits mitochondrial permeability transition. Circulation 2005, 111:194-197.

11. Wang L, Oka N, Tropak M, Callahan J, Lee J, Wilson G, Redington A, Caldarone CA: Remote ischemic preconditioning elaborates a transferable blood-borne effector that protects mitochondrial structure and function and preserves myocardial performance after neonatal cardioplegic arrest. J Thorac Cardiovasc Surg 2008, 136:335-342.

12. Heusch G, Boengler K, Schulz R: Inhibition of mitochondrial permeability transition pore opening: the Holy Grail of cardioprotection. Basic Res Cardiol 2010, 105:151-154.

13. Liu L, Zhu J, Brink PR, Glass PSA, Rebecchi MJ: Age-associated differences in the inhibition of mitochondrial permeability transition pore opening by cyclosporine A. Acta Anaesthesiol Scand 2011, 55:622-630.

14. Ludman AJ, Yellon DM, Hausenloy DJ: Cardiac preconditioning for ischaemia: lost in translation. Dis Model Mech 2010, 3:35-38.

15. Pfeffer MA, Pfeffer JM, Fishbein MC, Fletcher PJ, Spadaro J, Kloner RA, Braunwald E: Myocardial infarct size and ventricular function in rats. Circ Res 1979, 44:503-512.

16. Passonneau JV, Lauderdale VR: A comparison of three methods of glycogen measurement in tissues. Anal Biochem 1974, 60:405-412.

17. Bergmeyer H-U, Bernt E: Lactate dehydrogenase. In Methods of Enzymatic Analysis. Edited by Bergmeyer H-U. London: Academic; 1963:574-579.

18. Pasdois $P$, Beauvoit $B$, Costa ADT, Vinassa B, Tariosse L, Bonoron-Adèle $S$, Garlid KD, Santos PD: Sarcoplasmic ATP-sensitive potassium channel blocker HMR1098 protects the ischemic heart: Implication of calcium, complex I, reactive oxygen species and mitochondrial ATP-sensitive potassium channel. J Mol Cell Cardiol 2007, 42:631-642.

19. Di Lisa F, Bernardi P: Mitochondria and ischemia-reperfusion injury of the heart: Fixing a hole. Cardiovasc Res 2006, 70:191-199.

20. Baines CP: The mitochondrial permeability transition pore and ischemia-reperfusion injury. Basic Res Cardiol 2009, 104:181-188.

21. Weiss JN, Korge P, Honda HM, Ping P: Role of the mitochondrial permeability transition in myocardial disease. Circ Res 2003, 93:292-301.

22. Javadov SA, Clarke S, Das M, Griffiths EJ, Lim KH, Halestrap AP: Ischaemic preconditioning inhibits opening of mitochondrial permeability transition pores in the reperfused rat heart. J Physiol 2003, 549:513-524

23. Griffiths EJ, Halestrap AP: Protection by cyclosporin A of ischemia/ reperfusion-induced damage in isolated rat hearts. J Mol Cell Cardiol 1993, 25:1461-1469 
24. Clarke SJ, McStay GP, Halestrap AP: Sanglifehrin A acts as a potent inhibitor of the mitochondrial permeability transition and reperfusion injury of the heart by binding to cyclophilin-D at a different site from cyclosporin A. J Biol Chem 2002, 277:34793-34799.

25. Onishi A, Miyamae M, Kaneda K, Kotani J, Figueredo VM: Direct evidence for inhibition of mitochondrial permeability transition pore opening by sevoflurane preconditioning in cardiomyocytes: Comparison with cyclosporine A. Eur J Pharmacol 2012, 675:40-46.

26. Sharov VG, Todor A, Khanal S, Imai M, Sabbah HN: Cyclosporine A attenuates mitochondrial permeability transition and improves mitochondrial respiratory function in cardiomyocytes isolated from dogs with heart failure. J Mol Cell Cardiol 2007, 42:150-158.

27. Weinbrenner C, Liu GS, Downey JM, Cohen MV: Cyclosporine A limits myocardial infarct size even when administered after onset of ischemia. Cardiovasc Res 1998, 38:676-684.

28. Cross HR, Opie LH, Radda GK, Clarke K: Is a high glycogen content beneficial or detrimental to the ischemic rat heart? : A controversy resolved. Circ Res 1996, 78:482-491.

29. Halestrap AP, Pasdois $P$ : The role of the mitochondrial permeability transition pore in heart disease. Biochim Biophys Acta 2009, 1787:1402-1415.

30. Pasdois P, Parker JE, Halestrap AP: Extent of mitochondrial hexokinase II dissociation during ischemia correlates with mitochondrial cytochrome $c$ release, reactive oxygen species production, and infarct size on reperfusion. J Am Heart Assoc 2013, 2:e005645.

31. Pastorino J, Hoek J: Regulation of hexokinase binding to VDAC. J Bioenerg Biomembr 2008, 40:171-182.

32. Chiara F, Castellaro D, Marin O, Petronilli V, Brusilow WS, Juhaszova M, Sollott SJ, Forte M, Bernardi P, Rasola A: Hexokinase II detachment from mitochondria triggers apoptosis through the permeability transition pore independent of voltage-dependent anion channels. PLoS One 2008, 3:e1852.

33. Ferdinandy P, Schulz R, Baxter GF: Interaction of cardiovascular risk factors with myocardial ischemia/reperfusion injury, preconditioning, and postconditioning. Pharmacol Rev 2007, 59:418-458.

34. Balakumar $P$, Singh $H$, Singh M, Anand-Srivastava MB: The impairment of preconditioning-mediated cardioprotection in pathological conditions. Pharmacol Res 2009, 60:18-23.

35. Gaasch WH, Zile MR, Hoshino PK, Weinberg EO, Rhodes DR, Apstein CS: Tolerance of the hypertrophic heart to ischemia: Studies in compensated and failing dog hearts with pressure overload hypertrophy. Circulation 1990, 81:1644-1653.

36. Andersen A, Povlsen JA, Bøtker HE, Nielsen-Kudsk JE: Right ventricular hypertrophy and failure abolish cardioprotection by ischaemic pre-conditioning. Eur J Heart Fail 2013, 15:1208-1214.

37. Lopaschuk GD, Ussher JR, Folmes CDL, Jaswal JS, Stanley WC: Myocardial fatty acid metabolism in health and disease. Physiol Rev 2010, 90:207-258.

38. Stanley WC, Recchia FA, Lopaschuk GD: Myocardial substrate metabolism in the normal and failing heart. Physiol Rev 2005, 85:1093-1129.

39. Jaswal JS, Keung W, Wang W, Ussher JR, Lopaschuk GD: Targeting fatty acid and carbohydrate oxidation - A novel therapeutic intervention in the ischemic and failing heart. Biochim Biophys Acta 1813, 2011:1333-1350.

doi:10.1186/1479-5876-12-139

Cite this article as: Khaliulin et al:: Clinically-relevant consecutive treatment with isoproterenol and adenosine protects the failing heart against ischaemia and reperfusion. Journal of Translational Medicine 2014 12:139.

\section{Submit your next manuscript to BioMed Central and take full advantage of:}

- Convenient online submission

- Thorough peer review

- No space constraints or color figure charges

- Immediate publication on acceptance

- Inclusion in PubMed, CAS, Scopus and Google Scholar

- Research which is freely available for redistribution 